\title{
Pretreatment with the $\gamma$-secretase inhibitor DAPT sensitizes drug-resistant ovarian cancer cells to cisplatin by downregulation of Notch signaling
}

\author{
MINGYI WANG ${ }^{1}$, XIANGDONG MA ${ }^{2}$, JIAN WANG $^{2}$, LIN WANG $^{2}$ and YU WANG ${ }^{1}$ \\ ${ }^{1}$ Department of Obstetrics and Gynecology, General Hospital of the People's Liberation Army, \\ Chengdu Military Region; ${ }^{2}$ Department of Obstetrics and Gynecology, Xijing Hospital, \\ The Fourth Military Medical University, Xi'an, Shaanxi, P.R. China
}

Received December 8, 2013; Accepted January 30, 2014

DOI: 10.3892/ijo.2014.2301

\begin{abstract}
Notch signaling is implicated in ovarian cancer tumorigenesis and inhibition of Notch signaling with $\gamma$-secretase inhibitor DAPT resulted in reduction of tumor cell viability and induction of apoptosis in ovarian cancer cells. This study investigated whether DAPT has the same effect on ovarian cancer cells that are resistant to cisplatin and the underlying molecular events. Ovarian cancer cell lines resistant to cisplatin were treated with DAPT, cisplatin or combination for cell viability MTT, flow cytometric cell cycle, ELISA apoptosis and colony formation assays. qRT-PCR and western blotting were used to detect gene expressions. We found that pretreatment of ovarian cancer cisplatin-resistant cell lines with DAPT for $24 \mathrm{~h}$ and then with cisplatin for $72 \mathrm{~h}$ showed a synergistic antitumor activity in these cell lines, while cisplatin treatment and then addition of DAPT just showed an additive or antagonistic effects on these cisplatin-resistant ovarian cancer cells. Moreover, pretreatment of ovarian cancer cell lines with DAPT and then with cisplatin also inhibited tumor cell colony formation capacity, arrested tumor cells at $\mathrm{G} 2$ phase of the cell cycle and induced apoptosis. The cell cycle and apoptosis-related genes, such as cyclin B1, Bcl-2 and caspase-3, were also modulated by the treatment. Pretreatment of ovarian cancer cell lines with DAPT and then with cisplatin downregulated Notch1 and Hes1 expression dose- and time-dependently. The current data demonstrate that DAPT pretreatment was able to sensitize cisplatin-resistant human ovarian cancer cells to cisplatin by downregulation of Notch signaling.
\end{abstract}

Correspondence to: Professor Yu Wang, Department of Obstetrics and Gynecology, General Hospital of the People's Liberation Army, Chengdu Military Region 610036, P.R. China

E-mail: wangyuog@163.com

Key words: ovarian cancer, $\gamma$-secretase inhibitor, cisplatin-resistant cancer, Notch signaling, DAPT, Notch1, Hes1

\section{Introduction}

Ovarian cancer is the second most common gynecologic cancer in women and the leading cause of cancer death of gynecologic malignancy in the world (1). More than $90 \%$ ovarian cancers are believed to arise from the surface epithelium of the ovary and frequently absent of early signs and symptoms; thus most ovarian cancer patients are diagnosed at advanced stage of disease, which makes curable surgery infrequent and has a relatively poor prognosis. For past decades, chemotherapy has been a general standard of care for ovarian cancer with highly variable protocols and used alone or after surgery to treat any residual disease (2). Cisplatin-based chemotherapy is used as a primary treatment of ovarian cancer and its combination with other agents has become standard chemotherapy for treatment of advanced ovarian cancer, but prolonged drug treatment results in development of acquired drug resistance impeding successful treatment (3). The antineoplastic effect of cisplatin is mediated by formation of DNA adducts and inter- and intrastrand crosslinks (4). These adducts distort the DNA template with deceleration of cells in S phase followed with G2 phase arrest (5) and also result in diverse effects, including DNA synthesis inhibition, RNA transcription suppression, cell cycle arrest and apoptosis. Cisplatin resistance is multifactorial and rather complicated, including reduced platinum accumulation and enhanced platinum detoxification and metabolism in cells, altered DNA damage repair, activation of phospholipid kinase and phosphatidyl inositol 3-kinase and other signaling pathways ultimately causing dysregulation of apoptotic pathway (6). Thus, this disappointing outcome strongly suggests that a better understanding of the mechanisms of chemoresistance could lead to novel therapeutic strategies for effective control of ovarian cancer.

Our study is focusing on the Notch signaling. A previous study demonstrated that activation of the Notch signaling was linked to chemoresistance of pancreatic cancer to cisplatin (7) and inhibition of Notch signaling with $\gamma$-secretase inhibitors could sensitize colon cancer cells to chemotherapy and was synergistic with some antineoplastic agents (8). Indeed, the Notch signaling pathway plays a key role in the proliferation and differentiation of many tissues. This evolutionarily 
conserved pathway can regulate critical cell fate decision (9). In mammals, the Notch family consists four receptors (i.e., Notch1 to Notch4) and five ligands (Jagged-1, Jagged-2, Delta-like-1, Delta-like-3 and Delta-like-4) (10). Notch ligands and receptors are type I membrane proteins that regulate cell fate during cell-cell contact (10-12). Receptor-ligand interaction between two neighboring cells leads to $\gamma$-secretase-mediated proteolytic release of the Notch intracellular domain (NICD) (13). NICD then translocates into the nucleus, and in turn interacts with the transcriptional cofactor $\mathrm{CBF} 1$ and transactivates target genes, such as Hes and Hey families to affect numerous pathways involving cell-fate determination $(14,15)$. Abnormal Notch signaling has been documented in many cancers, including ovarian cancer $(16,17)$. Overexpression of Notch proteins was associated with poor prognosis of different cancer patients (18) and with tumor de-differentiation in ovarian cancer (19). Molecularly, activation of Notch proteins are triggered by $\gamma$-secretase, which cleaves the Notch receptor to activate the pathway (13). Previous studies showed that $\gamma$-secretase inhibitors were able to inhibit tumor cell viability and induced apoptosis in different cancer cell lines $(20,21)$. We found that $\gamma$-secretase inhibitor (DAPT)-blocked Notch signaling reduced viability of ovarian cancer A2780 cells but induced them to undergo apoptosis (22). In this study, we hypothesized that inhibition of Notch signaling by DAPT could sensitize drugresistant ovarian cancer cells to cisplatin chemotherapy. We assessed the effects of DAPT on sensitizing cisplatin-resistant ovarian cancer A2780/CP70 and OV2008/C13 cells to cisplatininduced cell death and the underling molecular events.

\section{Materials and methods}

Cell culture and reagents. Two pairs of cisplatin-sensitive and cisplatin-resistant human ovarian cancer cell lines A2780, A2780/CP70 and OV2008, OV2008/C13 were kindly provided by Dr Jun Hu (The Third Military Medical University, Chongqing, China) and maintained in RPMI-1640 medium containing $10 \%$ heat-inactivated fetal bovine serum (FBS; all from Xin Xing Tang Biotechnology Company, Beijing, China) at $37^{\circ} \mathrm{C}$ in a humidified $5 \% \mathrm{CO}_{2}$ atmosphere. For cisplatin treatment, cells were maintained in medium with the desired doses of cisplatin for $1 \mathrm{~h}$ and then washed with PBS and followed by incubation in fresh drug-free medium for varying times post-treatment.

Cell viability MTT assay. Cells $\left(5 \times 10^{3}\right)$ were seeded in 96-well cell culture plates, treated with different concentrations of $\gamma$-secretase inhibitor $\mathrm{N}$-[N-(3,5-difluorophenacetyl)-L-alanyl]S-phenylglycine t-butyl ester (DAPT), cisplatin (all from Sigma-Aldrich, St. Louis, MO, USA), or combination for 72 h. For combination experiments, ovarian cancer A2780/ CP70 and OV2008/C13 cells were treated for $72 \mathrm{~h}$ with DAPT (30 $\mu \mathrm{mol} / \mathrm{l}$ ) either $24 \mathrm{~h}$ before or after cisplatin ( 3 or $6 \mu \mathrm{mol} / \mathrm{l}$ ) treatment and then subjected to the MTT assay. Specifically, 3-(4,5-dimethylthiazol-2-yl)-2,5-diphenyltetrazolium bromide (MMT) was added to the cultures and the cells were incubated for an additional $4 \mathrm{~h}$. The resulting formazan crystals were solubilized by addition of $150 \mu$ l dimethyl sulfoxide (DMSO) to each well. The optical density was measured at $570 \mathrm{~nm}$ using a microplate reader and cell viability was determined by the formula: cell viability $(\%)=($ absorbance of the treated wells - absorbance of the blank control wells) / (absorbance of the negative control wells - absorbance of the blank control wells) x $100 \%$. All experiments were performed in triplicate and repeated at least three times. Drug interactions and isobologram were analyzed using CalcuSyn software (Biosoft, Beijing, China).

Colony formation assay. Ovarian cancer A2780/CP70 and OV2008/C13 cells $\left(5 \times 10^{4} / \mathrm{ml}\right)$ were seeded into 6-well plates according to the manufacturer's instructions. Cells were treated with DAPT $(30 \mu \mathrm{mol} / \mathrm{l})$ either $24 \mathrm{~h}$ before or after cisplatin ( 3 or $6 \mu \mathrm{mol} / \mathrm{l})$ treatment. After 14 days of incubation at $37^{\circ} \mathrm{C}$ in a humidified atmosphere containing $5 \% \mathrm{CO}_{2}$ in air, colonies were counted using an inverted microscope (Leica, Heidelberg, Germany).

Flow cytometric cell cycle assay. The cell cycle was analyzed using flow cytometry. Briefly, cells $\left(1 \times 10^{6}\right)$ were collected and washed in phosphate-buffered saline (PBS), then fixed in $75 \%$ ice-cold alcohol for $30 \mathrm{~min}$ at $4^{\circ} \mathrm{C}$. After washing with ice-cold PBS three times, cells were resuspended in $1 \mathrm{ml}$ of PBS containing $40 \mu \mathrm{g}$ of propidium iodide (Sigma-Aldrich) and $100 \mu \mathrm{g}$ of RNase A (Sigma-Aldrich) and incubated for $30 \mathrm{~min}$ at $37^{\circ} \mathrm{C}$. Samples were then analyzed by FACS (BD Immunocytometry Systems, San Jose, CA, USA). Each experiment was repeated for at least three times.

ELISA apoptosis assay. A Cell Death Detection ELISA kit (Roche, Shanghai, China) was used to detect apoptosis in treated cells according to the protocol provided by the manufacturer. Briefly, cell culture supernatants were washed away to remove fragmented DNA from necrotic cells, and then cells were lysed and loaded into microtiter plate modules coated with an anti-histone antibody for incubation for $45 \mathrm{~min}$ at room temperature. Next, samples were incubated with the anti-DNA peroxidase followed by color development with ABTS substrate. After that, the absorbance rates of these samples were measured using a microplate reader (SLT, Spectra LabInstruments Deutschland $\mathrm{GmbH}$ ) at 405 and $490 \mathrm{~nm}$ (reference wavelength).

RNA isolation and quantitative RT-PCR. Total RNA was isolated from ovarian cancer cells using the TRIzol reagent (Invitrogen, Carlsbad, CA, USA) according to the manufacturer's instructions and then reverse transcribed into cDNA using an PrimeScript ${ }^{\mathrm{TM}}$ RT reagent kit (Takara, Dalian, China). These cDNA samples were then amplified in the ABI 7500 system (Applied Biosystems) using SYBR Premix Ex Taq $^{\mathrm{TM}}$ II (Takara). Primers used for Hes1 were 5'-TGGAAAT GACAGTGAAGCACCTC-3' and 5'-TCGTTCATGCACTC GCTGAAG-3'. The internal control $\beta$-actin primers were 5'-TGGCACCCAGCACAATGAA-3' and 5'-CTAAGTCATAG TCCGCCTAGAAGCA-3'. Thermocycling was set as follows: $94^{\circ} \mathrm{C}$ for $5 \mathrm{~min}, 40$ cycles of $94^{\circ} \mathrm{C}$ for $30 \mathrm{~min}, 55^{\circ} \mathrm{C}$ for $30 \mathrm{~min}$ and $72^{\circ} \mathrm{C}$ for $60 \mathrm{~min}$, and a final extension at $72^{\circ} \mathrm{C}$ for $10 \mathrm{~min}$ and then permanently stored at $4^{\circ} \mathrm{C}$. Relative quantitation of mRNA expression levels was determined using the relative standard curve method according to the manufacturer's instructions (Applied Biosystems). 

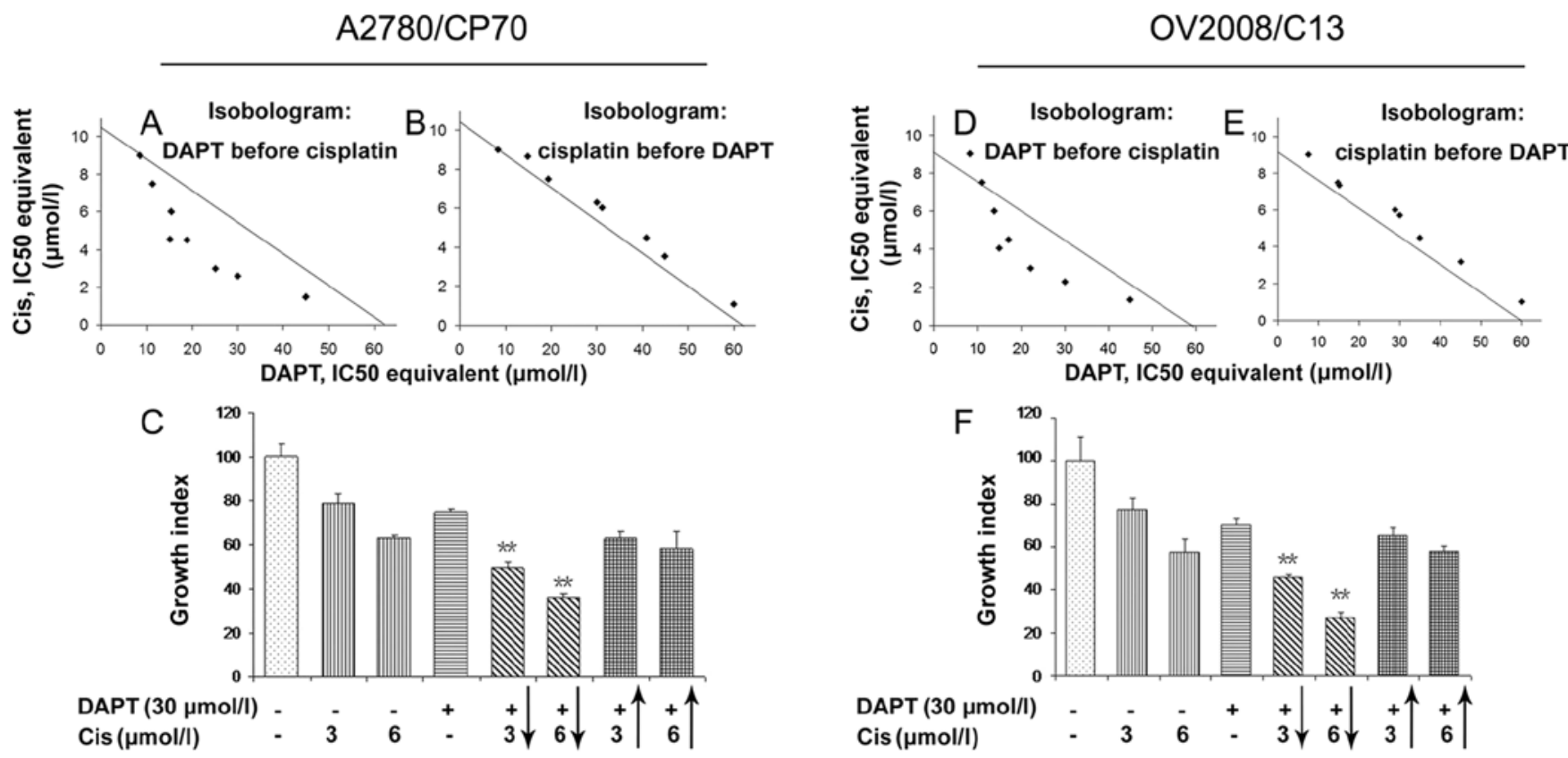

Figure 1. Synergistic effects of DAPT-cisplatin combination on ovarian cancer cells in a drug administration sequence. (A) MTT assay. Ovarian cancer A2780/ CP70 cells were treated with various concentrations of cisplatin and DAPT under the drug administration sequence: an initial 24-h DAPT exposure followed by 72-h cisplatin treatment. (B) MTT assay. A2780/CP70 cells were treated with different concentrations of cisplatin and DAPT under the drug administration sequence: an initial 24-h cisplatin exposure followed by 72-h DAPT treatment. $\mathrm{IC}_{50}$ and isobologram were calculated and processed using CalcuSyn software. (C) MTT assay. Cells were treated with DAPT 24 h before cisplatin (columns 5 and 6) or with cisplatin 24 h before DAPT (columns 7 and 8 ) as indicated by an arrow. (D) Ovarian cancer OV2008/C13 cells were treated with different concentrations of cisplatin and DAPT under the drug administration sequence: an initial 24-h DAPT exposure followed by 72-h cisplatin treatment. (E) MTT assay. OV2008/C13 cells were treated with different concentrations of cisplatin and DAPT under the drug administration sequence: an initial 24-h cisplatin exposure followed by 72 -h DAPT treatment. IC 50 and isobologram were calculated and processed using CalcuSyn software. (F) MTT assay. Cells were treated with DAPT for 24 h before cisplatin (columns 5 and 6 ) or with cisplatin $24 \mathrm{~h}$ before DAPT (columns 7 and 8) as indicated by an arrow. ${ }^{* *} \mathrm{P}<0.001$ compared to the corresponding single treatment using the Tukey-Kramer Multiple Comparison test $(n=3)$.

Protein extraction and western blot analysis. Total proteins from ovarian cancer A2780/CP70 and OV2008/C13 cells were lysed in a lysis buffer containing $\mathrm{NaCl}$, sodium desoxycholate, sodium dodecyl sulfate and Tris and incubated at $4^{\circ} \mathrm{C}$ for $15 \mathrm{~min}$. The protein concentrations were determined using the Bio-Rad assay system (Bio-Rad, Hercules, CA, USA). These protein samples were then fractionated using sodium dodecyl sulfate polyacrylamide (10\%) gels for electrophoresis (SDS-PAGE) and then transferred onto a nitrocellulose membrane (Kenker, USA). For western blotting, the membranes were blocked in 5\% non-fat milk in Tris-buffered saline containing $0.1 \%$ Tween-20 (TBS-T) and then incubated with appropriate primary antibodies overnight at $4^{\circ} \mathrm{C}$. Horseradish peroxidaseconjugated anti-goat IgG was used as the secondary antibody, and the protein bands were visualized using the enhanced chemiluminescence (ECL) method (GE Healthcare, USA) and quantified by using laser densitometry. The data were summarized as the mean of 3 independent experiments with the standard deviation. The membranes were then stripped by incubated for $30 \mathrm{~min}$ at $50^{\circ} \mathrm{C}$ in a buffer that contained $2 \%$ SDS, $62.5 \mathrm{mmol} / 1$ Tris (pH 6.7), and $100 \mathrm{mmol} / 1$ 2-mercaptoethanol and further washed and incubated with the desired primary antibody. Antibodies against Hes1, cyclin B1, caspase-3, Bcl-2, and $\beta$-actin were purchased from Santa Cruz Biotechnology (Santa Cruz, CA, USA).

Statistical analysis. The data were expressed as means \pm standard error. The Student's t-test was performed to analyze the data between groups. A P-value $<0.05$ was considered as statistically significant.

\section{Results}

DAPT potentiates cisplatin-reduced viability of ovarian cancer cells in a drug sequence-dependent manner. In this study, we first determined whether $\gamma$-secretase inhibitor DAPT was able to sensitize ovarian cancer cells to low-dose cisplatin-reduced cell viability. We treated A2780/CP70 and OV2008/C13 cells with various concentrations of DAPT up to $90 \mu \mathrm{mol} / \mathrm{l}$ and cisplatin up to $9 \mu \mathrm{mol} / 1$ under two different drug administration scenarios, i.e., an initial 24-h DAPT exposure followed by 72-h cisplatin treatment or vice versa. Cell viability was assessed and the values of inhibiting concentration (IC), were calculated. As shown in Fig. 1A and D, if DAPT was administered before cisplatin, DAPT can synergistically sensitize cisplatin antitumor activity in cisplatin-resistant ovarian cancer cell lines, i.e., most of $\mathrm{IC}_{50}$ were below the combination-isobol line. On the other hand, if DAPT was administered after cisplatin, DAPT was additive or antagonistic rather than synergistic effects with cisplatin, i.e., most of $\mathrm{IC}_{50}$ were above the combination-isobol line (Fig. 1B and E). After that, we selected two cisplatin doses (3 and $6 \mu \mathrm{mol} / \mathrm{l}$ ) that kill $\sim 20-40 \%$ of ovarian cancer cells and combined them with $30 \mu \mathrm{mol} / 1$ DAPT. Data showed that pretreatment of tumor cells with DAPT increased the potency of cisplatin-reduced cell viability, e.g., treatment of tumor cells with 3 and $6 \mu \mathrm{mol} / 1$ cisplatin for $72 \mathrm{~h}$ caused $\sim 21$ and 
Table I. Reduced cell viability (\%) by DAPT pretreatment and then cisplatin addition.

\begin{tabular}{|c|c|c|c|c|c|c|c|c|}
\hline \multirow[b]{2}{*}{$\mathrm{Cis}(\mu \mathrm{mol} / \mathrm{l})$} & \multicolumn{8}{|c|}{ DAPT $(\mu \mathrm{mol} / \mathrm{l})$} \\
\hline & 0 & 15 & 30 & 45 & 60 & 75 & 90 & $\mathrm{IC}_{50}$ \\
\hline 0 & 0 & 13 & 25 & 35 & 50 & 57 & 65 & 61.88 \\
\hline 1.5 & 15 & 31 & 42 & 55 & 62 & 67 & 70 & 36.93 \\
\hline 3 & 21 & 38 & 51 & 67 & 73 & 77 & 81 & 24.97 \\
\hline 4.5 & 27 & 46 & 58 & 75 & 78 & 83 & 86 & 18.79 \\
\hline 6 & 38 & 52 & 64 & 82 & 86 & 89 & 90 & 15.28 \\
\hline 7.5 & 43 & 61 & 73 & 88 & 89 & 92 & 93 & 11.13 \\
\hline 9 & 49 & 70 & 81 & 91 & 92 & 94 & 96 & 8.33 \\
\hline $\mathrm{IC}_{50}$ & 10.39 & 4.56 & 2.58 & 1.47 & 1.09 & 0.93 & 0.85 & \\
\hline
\end{tabular}

Bold text indicates a significant change after treatment.

Table II. Reduced cell viability (\%) by cisplatin pretreatment and then DAPT addition.

\begin{tabular}{|c|c|c|c|c|c|c|c|c|}
\hline \multirow[b]{2}{*}{ Cis $(\mu \mathrm{mol} / \mathrm{l})$} & \multicolumn{8}{|c|}{ DAPT $(\mu \mathrm{mol} / \mathrm{l})$} \\
\hline & 0 & 15 & 30 & 45 & 60 & 75 & 90 & $\mathrm{IC}_{50}$ \\
\hline 0 & 0 & 13 & 25 & 35 & 50 & 57 & 65 & 61.88 \\
\hline 1.5 & 15 & 22 & 30 & 38 & 54 & 59 & 66 & 55.61 \\
\hline 3 & 21 & 28 & 37 & 44 & 55 & 60 & 67 & 48.33 \\
\hline 4.5 & 27 & 32 & 41 & 56 & 61 & 63 & 69 & 40.83 \\
\hline 6 & 38 & 39 & 42 & 59 & 63 & 67 & 72 & 31.25 \\
\hline 7.5 & 43 & 48 & 54 & 62 & 65 & 68 & 73 & 19.41 \\
\hline 9 & 49 & 57 & 63 & 64 & 66 & 70 & 75 & 8.32 \\
\hline $\mathrm{IC}_{50}$ & 10.39 & 8.65 & 6.27 & 3.51 & 1.09 & 0.53 & 0.13 & \\
\hline
\end{tabular}

Bold text indicates a significant change after treatment.

$38 \%$ reduction of viability of A2780/CP70 cells, respectively. In contrast, tumor cell viability was reduced to 51 and $64 \%$ when pretreated with DAPT (Fig. 1C and F and Table I), whereas tumor cell viability was only reduced to 37 and $42 \%$ for 3 and $6 \mu \mathrm{mol} / \mathrm{l}$ cisplatin, respectively, if cisplatin was administrated before DAPT (Table II).

Furthermore, we also determined the effects of their combination in regulation of tumor cell colony formation capacity. Our data showed that pretreatment of tumor cells with DAPT increased the potency of cisplatin-reduced colony formation in vitro (Fig. 2).

Combination of DAPT with cisplatin arrests cisplatinresistant ovarian cancer cells in $G 2$ phase of cell cycle. We next assessed the changed cell cycle after their treatment. In untreated control cells, the percentage of cells in $\mathrm{G} 1, \mathrm{~S}$, and $\mathrm{G} 2$ phases were $65.42,22.73$ and $11.85 \%$, respectively, while $3 \mu \mathrm{mol} / 1$ cisplatin treatment had no significant effect on changes in the cell cycle distributions, whereas $30 \mu \mathrm{mol} / 1$ DAPT alone caused cell cycle redistribution to G2 phase. In contrast, DAPT-potentiated cisplatin treatment at the above named dose resulted in a pronounced $\mathrm{G} 2$ arrest (\% of G1, S, and $\mathrm{G} 2$ phase cells was $18.90,21.85$ and $59.25 \%$, respectively). We also found a high proportion of sub-G1 phase popula- tion (apoptotic cells) in the DAPT-cisplatin treated tumor cells (Fig. 3A and B). Molecularly, DAPT-cisplatin treatment reduced the levels of cyclin B1 protein (Fig. 3C).

DAPT enhances cisplatin-induced apoptosis in ovarian cancer cells. Since DAPT combination with low dose of cisplatin had a high proportion of sub-G1 phase population, we determined apoptosis levels in A2780/CP70 cells. As shown in Fig. 4A, treatment of A2780/CP70 cells with $30 \mu \mathrm{mol} / 1 \mathrm{DAPT}$ or 3 or $6 \mu \mathrm{mol} / 1$ cisplatin caused negligible increase in tumor cell apoptosis over the background. However, pretreatment of tumor cells with DAPT for $24 \mathrm{~h}$ and then with cisplatin for $72 \mathrm{~h}$ caused a significant increase in apoptosis (Fig. 4A, columns 5 and 6), whereas cisplatin treatment before DAPT addition at the same dose did not show a significant increase in cell death (Fig. 4A, columns 7 and 8). These findings further confirmed that the combination of DAPT with low dose of cisplatin resulted in induction of apoptosis in A2780/CP70 cells in a drug sequence-dependent manner. At the molecular level, expression of apoptosis-related genes, such as caspase-3 and Bcl-2 proteins was also altered, i.e., caspase-3 was significantly higher and Bcl-2 was lower in DAPT before cisplatin treatment (Fig. 4B, lanes 5 and 6), which were in accordance with the cell death data in Fig. 4A. 


\section{A2780/CP70}

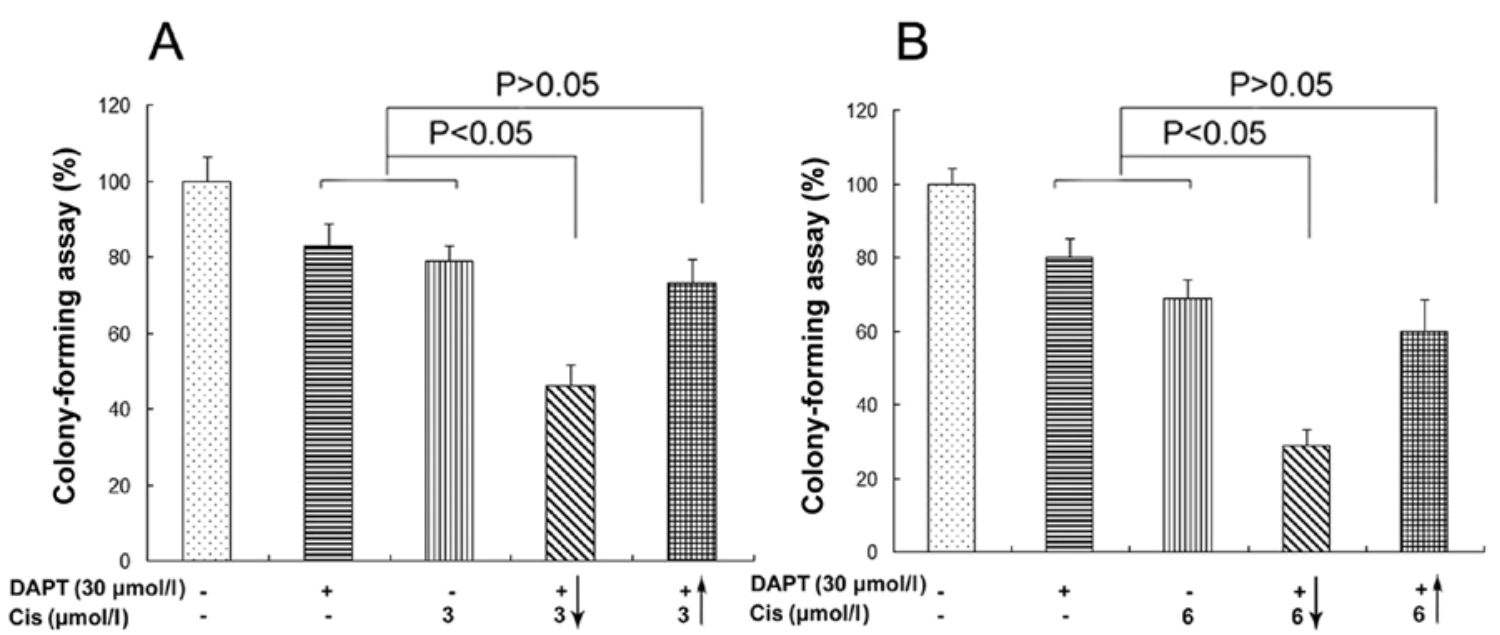

\section{OV2008/C13}

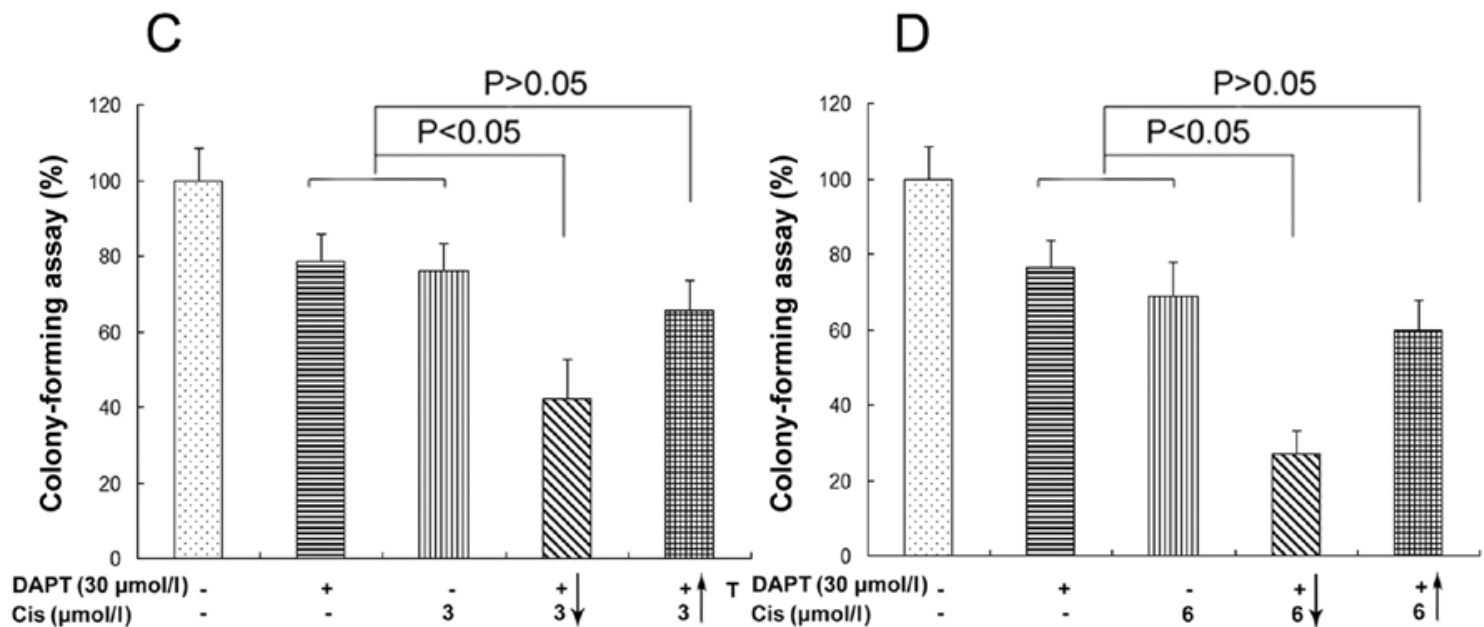

Figure 2. Effects of DAPT and cisplatin treatment on regulation of ovarian cancer cell colony formation capacity. (A) Cells treated with $3 \mu \mathrm{mol} / 1$ cisplatin and $30 \mu \mathrm{mol} / 1$ DAPT in A2780/CP70 cells. (B) Cells treated with $6 \mu \mathrm{mol} / 1$ cisplatin and $30 \mu \mathrm{mol} / 1 \mathrm{DAPT}$ in A2780/CP70 cells. (C) Cells treated with $3 \mu \mathrm{mol} / 1$ cisplatin and $30 \mu \mathrm{mol} / 1$ DAPT in OV2008/C13 cells. (D) Cells treated with $6 \mu \mathrm{mol} / 1$ cisplatin and $30 \mu \mathrm{mol} / 1$ DAPT in OV2008/C13 cells.

DAPT treatment downregulated Notch signaling and its target gene Hesl expression in ovarian cancer cells. We investigated potential targets of DAPT in ovarian cancer cells. qRT-PCR data revealed that different concentrations of DAPT (30 or $45 \mu \mathrm{mol} / 1)$ resulted in significant downregulation of Notch1 mRNA (Fig. 5A and B) and western blot analysis showed that levels of Notch1 protein were also downregulated by DAPT treatment in A2780/CP70 and OV2008/C13 cells (Fig. 5A and $\mathrm{B})$. These findings indicated that the Notch1 signaling pathway was efficiently suppressed by DAPT treatment in A2780/CP70 and OV2008/C13 cells in a dose-dependent manner. The concentration of $30 \mu \mathrm{M}$ was therefore used subsequently to effectively inhibit the Notch1 pathway.

To further determine if DAPT could downregulate expression of Notch1 downstream gene Hes1, we performed qRT-PCR and western blot analyses and found that different concentrations of DAPT (30 and $45 \mu \mathrm{mol} / \mathrm{l}$ ) significantly inhibited levels of Hes1 mRNA and protein (Fig. 5C and D). These findings indicated that DAPT treatment downregulated expression of Hes1 dose-dependently.

Moreover, we also performed time course treatments for changed expression of these genes. Our data showed that the altered expression of Notch1 was observed as early as $6 \mathrm{~h}$ after DAPT $(30 \mu \mathrm{mol} / \mathrm{l})$ treatment and was more pronounced with a longer period of treatment in A2780/CP70 and OV2008/C13 cells (Fig. 5E and F). These data suggested that the Notch1 signaling pathway was efficiently blocked by DAPT treatment in A2780/CP70 and OV2008/C13 cells in a time-dependent manner. Similarly, the altered expression of Hes1 gene was observed as early as $6 \mathrm{~h}$ after DAPT $(30 \mu \mathrm{mol} / \mathrm{l})$ treatment and was more pronounced with a longer period of treatment in A2780/CP70 and OV2008/C13 cells (Fig. 5G and H). 

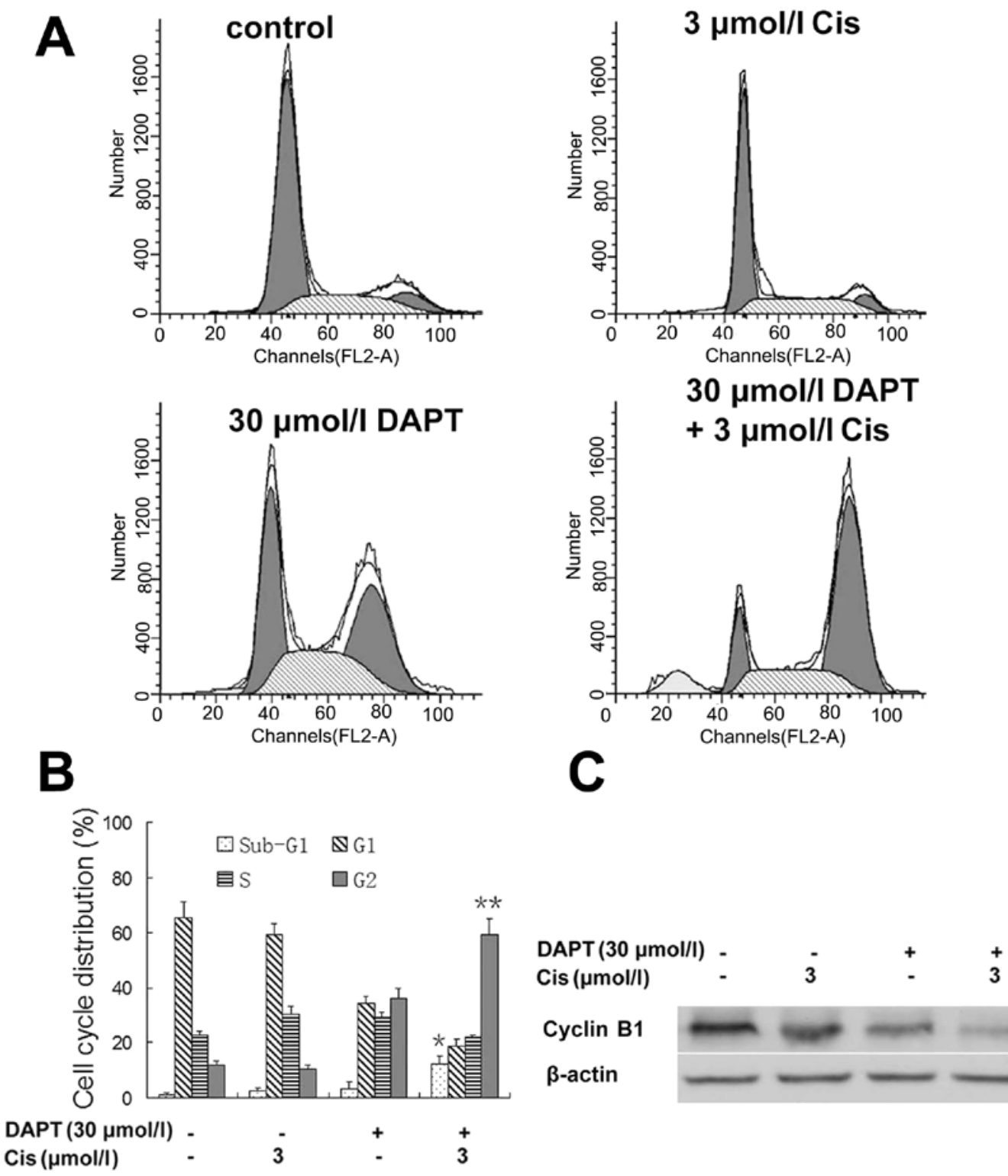

Cyclin B1

$\boldsymbol{\beta}$-actin

Figure 3. Effects of DAPT and cisplatin treatment on regulation of ovarian cancer cell cycle distribution. (A and B) A2780/CP70 cells were treated with $30 \mu \mathrm{mol} / 1$ DAPT for $24 \mathrm{~h}$, followed by treatment with $3 \mu \mathrm{mol} / 1$ cisplatin for another $72 \mathrm{~h}$. The cell cycle distribution was analyzed by using flow cytometry. ${ }^{*} \mathrm{P}<0.05 ;{ }^{* *} \mathrm{P}<0.01$ compared to the single treatment groups by the Tukey-Kramer Multiple Comparison test $(\mathrm{n}=3)$. (C) Western blotting. The duplicated cells were subjected to western blot analysis of cyclin B1 expression.

A

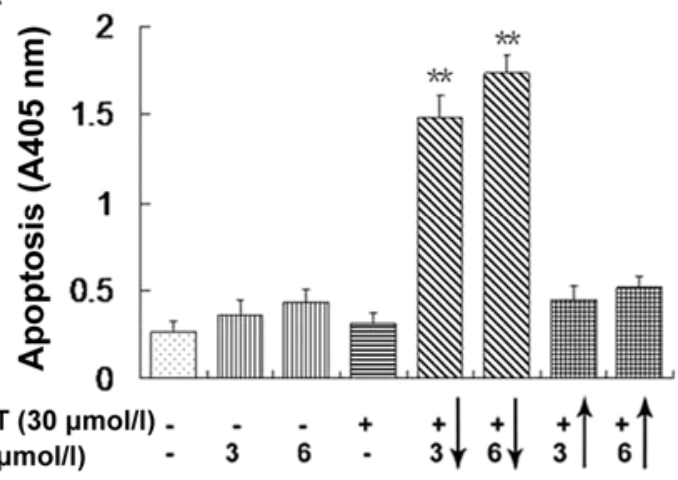

B

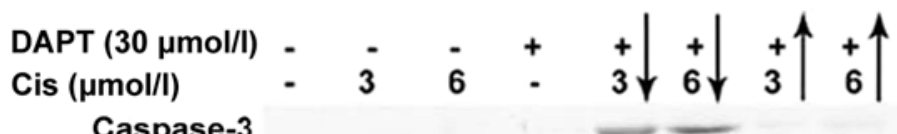

Caspase-3

Bcl-2

$\beta$-actin

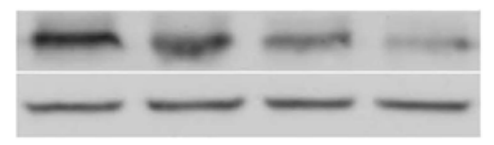



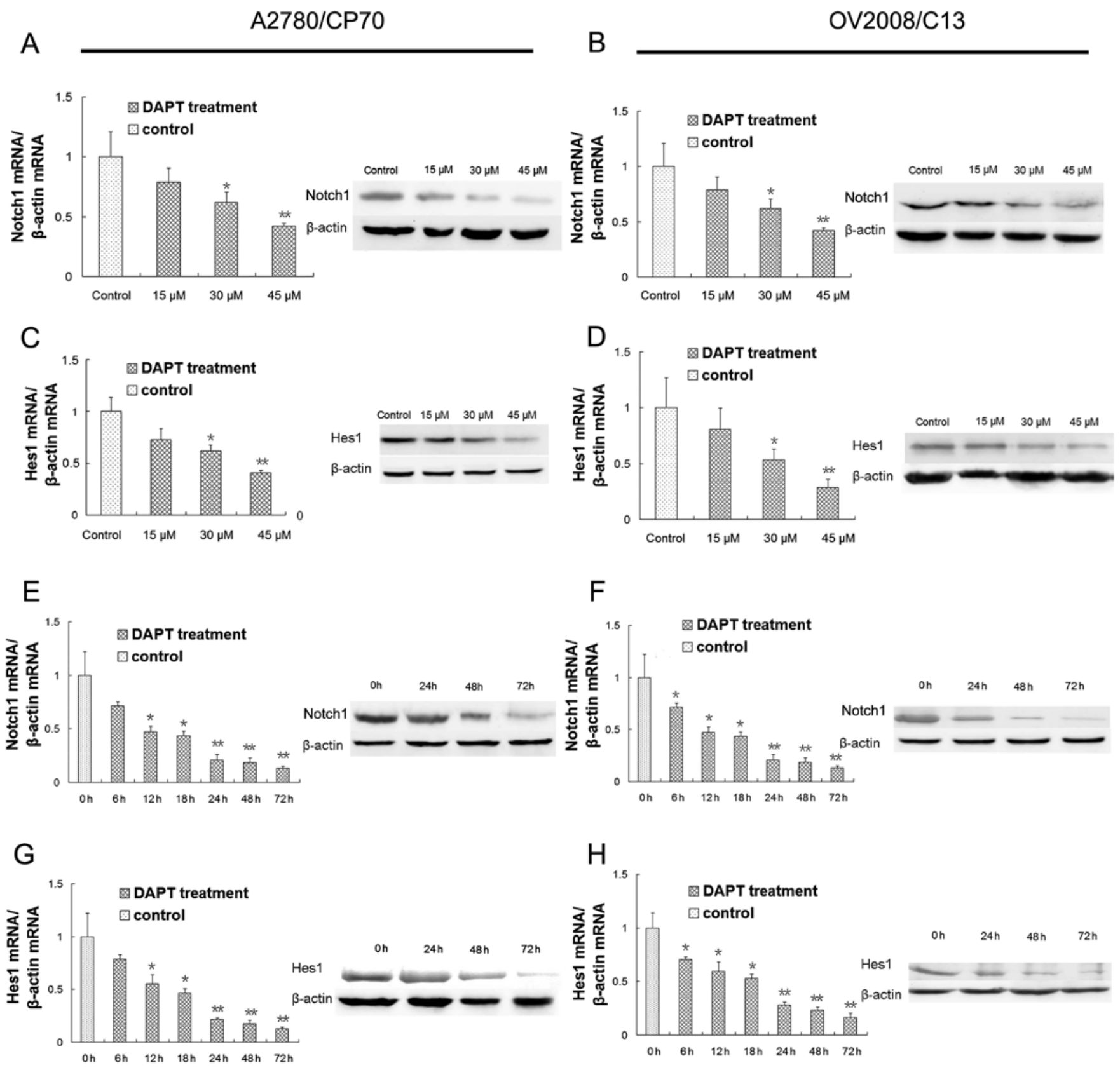

Figure 5. Effects of DAPT and cisplatin on regulation of gene expression. (A) Inhibition of Notch1 mRNA and protein expression after $72 \mathrm{~h}$ DAPT treatment in ovarian cancer cisplatin-resistant A2780/CP70 cells. (B) Inhibition of Notch1 mRNA and protein expression after $72 \mathrm{~h}$ DAPT treatment in ovarian cancer cisplatin-resistant OV2008/C13 cells. (C) Inhibition of Hes1 mRNA and protein expression after $72 \mathrm{~h}$ DAPT treatment in A2780/CP70 cells. (D) Inhibition of Hes1 mRNA and protein expression after $72 \mathrm{~h}$ DAPT treatment in OV2008/C13 cells. (E) Inhibition of Notch1 mRNA after 6, 12, 18, 24, 48 and $72 \mathrm{~h}$ and protein expression after 24, 48 and $72 \mathrm{~h}$ with $30 \mu \mathrm{M} / 1$ DAPT treatment in A2780/CP70 cells. (F) Inhibition of Notch1 mRNA after 6, 12, 18, 24, 48 and 72 h and protein expression after 24,48 and $72 \mathrm{~h}$ with $30 \mu \mathrm{M} / 1$ DAPT treatment in OV2008/C13 cells. (G) Inhibition of Hes1 mRNA after 6, 12, 18, 24, 48 and $72 \mathrm{~h}$ and protein expression after 24, 48 and $72 \mathrm{~h}$ with $30 \mu \mathrm{M} / 1$ DAPT treatment in A2780/CP70 cells. (H) Inhibition of Hes1 mRNA after 6, 12, 18, 24, 48 and 72 h and protein expression after 24, 48 and $72 \mathrm{~h}$ with $30 \mu \mathrm{M} / 1$ DAPT treatment in OV2008/C13 cells. Control cells were treated with dimethyl sulfoxide (DMSO). ${ }^{*} \mathrm{P}<0.05 ;{ }^{* *} \mathrm{P}<0.01$ compared to the controls.

\section{Discussion}

Notch signaling is implicated in ovarian cancer tumorigenesis $(19,23)$. Our previous studies showed that inhibition of Notch signaling with $\gamma$-secretase inhibitor DAPT resulted in reduced tumor cell viability and induction of apoptosis in ovarian cancer cells (22). In this study, we assessed whether DAPT has the same effect on ovarian cancer cells that are resistant to cisplatin and the underlying molecular events. We found that pretreatment of ovarian cancer cell lines with DAPT and then with cisplatin can synergistically sensitize cisplatin antitumor activity in cisplatin-resistant ovarian cancer cell lines, while cisplatin treatment with a delayed DAPT treatment had an additive or antagonistic effects on these cisplatin-resistant ovarian cancer cells. Similarly, these treatments also inhibited tumor cell colony formation capacity, arrested tumor cells at G2 phase of the cell cycle, but induced apoptosis. Expression of the cell cycle-related gene cyclin B1 and apoptosis-related 
gene Bcl-2 was suppressed but apoptosis-related gene caspase-3 was activated by these treatments. Molecularly, pretreatment of ovarian cancer cell lines with DAPT and then with cisplatin downregulated Notch1 and Hes1 expression dose- and timedependently. This study demonstrated that DAPT pretreatment could sensitize cisplatin-resistant human ovarian cancer cells to cisplatin by downregulation of Notch signaling. Future in vivo study need to confirm our current data before translating into clinical trials.

To date, cisplatin is still widely used in the treatment of various human cancers, including testicular, ovarian, cervical, bladder, head and neck, esophageal and lung cancers (24). In spite of the efficacy of cisplatin-based treatment regimens, long-term cure is difficult to obtain due to drug resistance (3), although great efforts have been made to develop combining chemotherapeutic agents to potentiate the effectiveness of current cytostatic drugs and to overcome chemotherapy resistance (8). Thus, our present study could provide a novel strategy by using combined agents to treat ovarian cancer. Our data showed pretreatment of ovarian cancer cell lines with DAPT and then with cisplatin synergistically sensitized cisplatin antitumor activity in cisplatin-resistant ovarian cancer cell lines. Our treatment regime could reduce the toxic dose of cisplatin but achieved synergistic effects on ovarian cancer cells.

Indeed, $\gamma$-secretase is a critical proteinase for Notch protein activation via nicastrin ectodomain binding to the N-terminus of Notch protein and cleavage of NICD (25). Thus, $\gamma$-secretase inhibitors can prevent generation of the intracellular domain of Notch protein and suppress the Notch activity (26). Notch signaling is implicated in ovarian cancer tumorigenesis $(19,23)$. Recently, there has been increased enthusiasm in targeting this pathway using $\gamma$-secretase inhibitors for novel and effective cancer therapy strategy (27). For example, treatment of leukemia using this strategy revealed a better efficacy but less side effects of $\gamma$-secretase inhibitors (28). A $\gamma$-secretase inhibitor GSI could be helpful in treating human T-cell acute lymphoblastic leukemia (T-ALL) by inhibiting the Notch signaling (29) and combination therapy of $\gamma$-secretase inhibitors with glucocorticoids improved the anti-leukemic effects of $\gamma$-secretase inhibitors and reduced their gut toxicity in vivo (30). It has also been reported that combination of $\gamma$-secretase inhibitors with chemotherapy might represent a novel approach for treating metastatic colon cancers (8). In the present study, we investigated the potential use of DAPT to sensitize the cisplatin-resistant ovarian cancer A2780/CP70 and OV2008/C13 cells to cisplatin. We found that DAPT pretreatment reduced cisplatin-resistant ovarian cancer cells to low-dose cisplatin-induced cell death by downregulation of the Notch signaling.

Previous studies reported that DAPT had anti-proliferative activity against several human cancer cell lines $(22,31)$. This anti-proliferative effect was due to induction of apoptosis and cell cycle arrest. Molecularly, Bcl-2 and caspase-3 play an importance role in regulation of apoptosis $(32,33)$ and cyclin B1 is one of the key molecules in the cell cycle modulation (34). Thus, our present study revealed an accumulation of G2 cell cycle arrest after DAPT treatment and DAPT-cisplatin treatment in cisplatin-resistant human ovarian cancer A2780/ CP70 cells. As G2 arrest is typically linked to DNA damage response, we postulate that DAPT pretreatment-sensitized A2780/CP70 cells to respond to DNA damage. Moreover, overexpression of caspase-3 could sensitize breast cancer cells to drug-induced apoptosis and enhance chemosensitivity (35). In contrast, Bc1-2 expression can confer cisplatin-induced apoptosis in ovarian cancer cells (36). Our current data showed that inhibition of Notch signaling by DAPT sensitized cisplatin-resistant human ovarian cancer cells and enhances chemosensitivity associated with modulation of apoptosisrelated gene expression.

Nevertheless, $\gamma$-secretase inhibitors can cleave a number of proteins, such as ErbB-4, CD44, E-cadherin and Notch family proteins (37). In the present study, we observed that DAPT resulted in downregulation of Hes1 in ovarian cancer cells in a dose-dependent manner by confirmed DAPT-inhibited Notch expression. However, it is unknown whether there are other signaling pathways or other important events in this process and further study is needed. Apoptosis induced by cisplatin is another feature of cellular response to combined DAPT and cisplatin treatment, which manifests as the synergetic inhibitory effect on cisplatin-resistant ovarian cancer cells, although the underlying molecular mechanisms mediating this synergistic antitumor effect are not completely understood. Further studies are required to better understand how the combination of $\gamma$-secretase inhibitors with cisplatin treatment can sensitize cisplatin-resistant ovarian cancer cells, especially the sequential use of DAPT and cisplatin in combination for control of ovarian cancer.

\section{Acknowledgements}

We thank Medjaden Bioscience Limited, Hong Kong, China, for assistance in preparation of this manuscript.

\section{References}

1. Siegel R, Naishadham D and Jemal A: Cancer statistics, 2013. CA Cancer J Clin 63: 11-30, 2013.

2. McGuire WP III and Markman M: Primary ovarian cancer chemotherapy: current standards of care. Br J Cancer 89 (Suppl 3): S3-S8, 2003.

3. Stewart JJ, White JT, Yan X, et al: Proteins associated with Cisplatin resistance in ovarian cancer cells identified by quantitative proteomic technology and integrated with mRNA expression levels. Mol Cell Proteomics 5: 433-443, 2006.

4. Mellish KJ, Barnard CF, Murrer BA and Kelland LR: DNA-binding properties of novel cis- and trans platinum-based anticancer agents in 2 human ovarian carcinoma cell lines. Int $\mathbf{J}$ Cancer 62: 717-723, 1995.

5. O'Neill CF, Koberle B, Masters JR and Kelland LR: Genespecific repair of $\mathrm{Pt} / \mathrm{DNA}$ lesions and induction of apoptosis by the oral platinum drug JM216 in three human ovarian carcinoma cell lines sensitive and resistant to cisplatin. Br J Cancer 81: 1294-1303, 1999.

6. Ohmichi M, Hayakawa J, Tasaka K, Kurachi H and Murata Y: Mechanisms of platinum drug resistance. Trends Pharmacol Sci 26: 113-116, 2005.

7. Wang Z, Li Y, Kong D, et al: Acquisition of epithelial-mesenchymal transition phenotype of gemcitabine-resistant pancreatic cancer cells is linked with activation of the notch signaling pathway. Cancer Res 69: 2400-2407, 2009.

8. Meng RD, Shelton CC, Li YM, et al: gamma-Secretase inhibitors abrogate oxaliplatin-induced activation of the Notch-1 signaling pathway in colon cancer cells resulting in enhanced chemosensitivity. Cancer Res 69: 573-582, 2009.

9. Kuroda Y, Murakami N, Morota M, et al: Impact of concurrent chemotherapy on definitive radiotherapy for women with FIGO IIIb cervical cancer. J Radiat Res 53: 588-593, 2012. 
10. Lai EC: Notch signaling: control of cell communication and cell fate. Development 131: 965-973, 2004.

11. Artavanis-Tsakonas S, Rand MD and Lake RJ: Notch signaling: cell fate control and signal integration in development. Science 284: 770-776, 1999.

12. Nickoloff BJ, Osborne BA and Miele L: Notch signaling as a therapeutic target in cancer: a new approach to the development of cell fate modifying agents. Oncogene 22: 6598-6608, 2003

13. Suwannarurk K, Bhamarapravatana K, Thaweekul Y, Mairaing K, Poomtavorn Y and Pattaraarchachai J: A 1-year experience with liquid-based and conventional papanicolaou smear in Thammasat University Hospital. J Med Assoc Thai 94 (Suppl 7): S47-S51, 2011

14. Wagner U, Marth C, Largillier R, et al: Final overall survival results of phase III GCIG CALYPSO trial of pegylated liposomal doxorubicin and carboplatin vs paclitaxel and carboplatin in platinum-sensitive ovarian cancer patients. Br J Cancer 107: 588-591, 2012

15. Doll CM,Aquino-Parsons C,Pintilie M, et al: The significance of tumoral ERCC1 status in patients with locally advanced cervical cancer treated with chemoradiation therapy: a multicenter clinicopathologic analysis. Int J Radiat Oncol Biol Phys 85: 721-727, 2013.

16. Miyamura T, Masuzaki $\mathrm{H}$ and Ishimaru T: Conservative treatment of a cervical pregnancy with local methotrexate injection. Int J Gynaecol Obstet 45: 62-63, 1994.

17. Rose SL, Kunnimalaiyaan M, Drenzek J and Seiler N: Notch 1 signaling is active in ovarian cancer. Gynecol Oncol 117: 130-133, 2010 .

18. Chu D, Wang W, Xie H, et al: Notch1 expression in colorectal carcinoma determines tumor differentiation status. J Gastrointest Surg 13: 253-260, 2009.

19. Wang M, Wang J, Wang L, Wu L and Xin X: Notch1 expression correlates with tumor differentiation status in ovarian carcinoma. Med Oncol 27: 1329-1335, 2010

20. Yoo J, Choi JY, Moon SH, et al: Prognostic significance of volume-based metabolic parameters in uterine cervical cancer determined using $18 \mathrm{~F}$-fluorodeoxyglucose positron emission tomography. Int J Gynecol Cancer 22: 1226-1233, 2012.

21. Sorosky JI: Endometrial cancer. Obstet Gynecol 120: 383-397, 2012.

22. Wang M, Wu L, Wang L and Xin X: Down-regulation of Notch1 by gamma-secretase inhibition contributes to cell growth inhibition and apoptosis in ovarian cancer cells A2780. Biochem Biophys Res Commun 393: 144-149, 2010.

23. Hopfer O, Zwahlen D, Fey MF and Aebi S: The Notch pathway in ovarian carcinomas and adenomas. Br J Cancer 93: 709-718, 2005 .
24. Tsang RY, Al-Fayea T and Au HJ: Cisplatin overdose: toxicities and management. Drug Saf 32: 1109-1122, 2009.

25. Jones ME, van Leeuwen FE, Hoogendoorn WE, et al: Endometrial cancer survival after breast cancer in relation to tamoxifen treatment: pooled results from three countries. Breast Cancer Res 14: R91, 2012.

26. Ma K, Wen HW and Liao QP: Expression of Notch3 and Notch intracellular domain in ovarian carcinoma and effect of $\mathrm{N}-[\mathrm{N}-$ (3,5-difluorophenyl) acetyl-L-alanyl]-S-phenyl glycine t-butyl ester on ovarian carcinoma cell. Zhonghua Fu Chan Ke Za Zhi 45: 921-926, 2010 (In Chinese)

27. Ma XG, Wang YM, Xue FX, et al: Clinicalpathological characteristics of Lynch syndrome related epithelial ovarian cancer. Zhonghua Fu Chan Ke Za Zhi 47: 201-204, 2012 (In Chinese).

28. van den Akker T, Beltman J, Leyten J, et al: The WHO maternal near miss approach: consequences at Malawian District level. PLoS One 8: e54805, 2013.

29. Masuda S, Kumano K, Suzuki T, et al: Dual antitumor mechanisms of Notch signaling inhibitor in a T-cell acute lymphoblastic leukemia xenograft model. Cancer Sci 100 : 2444-2450, 2009.

30. Kohorn EI: Imaging practices in the diagnosis and management of gestational trophoblastic disease: an assessment. J Reprod Med 57: 207-210, 2012.

31. Sun XM, Wen HW, Chen CL and Liao QP: Expression of Notch intracellular domain in cervical cancer and effect of DAPT on cervical cancer cell. Zhonghua Fu Chan Ke Za Zhi 44: 369-373, 2009 (In Chinese).

32. Korsmeyer SJ: Regulators of cell death. Trends Genet 11: 101-105, 1995.

33. Drexler HC: Programmed cell death and the proteasome. Apoptosis 3: 1-7, 1998

34. Ito M: Factors controlling cyclin B expression. Plant Mol Biol 43 $677-690,2000$

35. Friedrich K, Wieder T, Von Haefen C, et al: Overexpression of caspase-3 restores sensitivity for drug-induced apoptosis in breast cancer cell lines with acquired drug resistance. Oncogene 20: 2749-2760, 2001

36. Williams J, Lucas PC, Griffith KA, et al: Expression of Bcl-xL in ovarian carcinoma is associated with chemoresistance and recurrent disease. Gynecol Oncol 96: 287-295, 2005.

37. Beel AJ and Sanders CR: Substrate specificity of gammasecretase and other intramembrane proteases. Cell Mol Life Sci 65: 1311-1334, 2008. 